\title{
Erratum to: Bound State of an Electron in a MOS Structure Due to the Spin-Orbit Interaction
}

\author{
M. M. Mahmoodian ${ }^{a, *}$ and A. V. Chaplik ${ }^{b, * *}$ \\ ${ }^{a}$ Rzhanov Institute of Semiconductor Physics, Siberian Branch, Russian Academy of Sciences, Novosibirsk, 630090 Russia \\ ${ }^{b}$ Novosibirsk State University, Novosibirsk, 630090 Russia \\ *e-mail:mahmood@isp.nsc.ru \\ **e-mail: chaplik@isp.nsc.ru \\ Received March 13, 2019
}

DOI: $10.1134 / \mathrm{S} 1063776119050170$

We found a mistake in our paper mentioned in the title of this note. Electric field normal to the structure $F$ is a sum of the constant part $\bar{F}$ and contribution from the electron-electron interaction $\tilde{F}$, depending on the interparticle separation $\boldsymbol{\rho}$. Equation (2) in the paper relates to only $F=\bar{F}$, but the spin-orbit interaction (SOI) depending on $\tilde{F}$ should be written in the form $A \tilde{F} \mathbf{n}\left[\left(\hat{\mathbf{p}}_{1}-\hat{\mathbf{p}}_{2}\right) \times\left(\boldsymbol{\sigma}_{1}-\boldsymbol{\sigma}_{2}\right)\right]$, as this energy depends on the electron velocity relative to the source of the field. But $\hat{\mathbf{p}}_{1}-\hat{\mathbf{p}}_{2}=2 \hat{\mathbf{p}}=-2 i \partial_{\boldsymbol{\rho}}$, and that is why in all of the following formulas $F$ should be understood as $\bar{F}+2 \tilde{F}$, while in the Eq. (19) in the terms with total momentum $P$ one has to put $F=\bar{F}$, where $\bar{F}$ is the field due to gate voltage. The fields of the own image charges of electrons do not contribute to SOI. With accounting for the above-mentioned changes, the electron pair bound energy for $P=0$ equals $\Delta \simeq$ $3.64 \mathrm{meV}$ for $\bar{F}=3.2 \times 10^{4} \mathrm{~V} / \mathrm{cm}$, and same values of the rest parameters that are given in the paper.

The original article can be found online at https://doi.org/10.1134/S1063776118120075 